\title{
In Silico Analysis of Single Nucleotide Polymorphisms (SNPs) in Human MPL Gene
}

\author{
Mohamed Mubarak Babeker ${ }^{1,2}$, Afra Mohamed Suliman Albakry ${ }^{1}$, \\ Mohammed Nagm Eldin Elsamani ${ }^{3}$, Gihan Mossalami ${ }^{2}$, Hind Abdelaziz Elnasri, \\ Mona Abdelrahman Mohamed Khaier ${ }^{1, *}$ \\ ${ }^{1}$ Department of Molecular Biology and Bioinformatics, University of Bahri, Khartoum, Sudan \\ ${ }^{2}$ Department of Haematology, Omdurman Ahlia University, Khartoum, Sudan \\ ${ }^{3}$ Department of Clinical Biochemistry, Nile College, Khartoum, Sudan
}

Email address:

munakhaier@gmail.com (M. A. M. Khaier)

${ }^{*}$ Corresponding author

\section{To cite this article:}

Mohamed Mubarak Babeker, Afra Mohamed Suliman Albakry, Mohammed Nagm EldinElsamani, Gihan Mossalami, Hind Abdelaziz Elnasri, Mona Abdelrahman Mohamed Khaier. In Silico Analysis of Single Nucleotide Polymorphisms (SNPs) in Human MPL Gene International Journal of Genetics and Genomics. Vol. 7, No. 4, 2019, pp. 124-131. doi: 10.11648/j.ijgg.20190704.17

Received: September 30, 2019; Accepted: October 23, 2019; Published: November 22, 2019

\begin{abstract}
Thrombopoietin was shown to be the major regulator of megakaryocytopoiesis and platelet formation. The protein encoded by the c-mpl gene, CD110, is a 635 amino acid transmembrane domain, with two extracellular cytokine receptor domains and two intracellular cytokine receptor box motifs. Mutations to this gene are associated with myelofibrosis and essential Thrombocythemia. In essential Thrombocythemia, these mutations lead to the production of Thrombopoietin receptors that are constitutively activated, or constantly turned on, which results in the overproduction of abnormal megakaryocytes. MPL gene was investigated in NCBI database (http://www.ncbi.nlm.nih.gov/) and computational software's analyzed SNPs. SNPs in the coding region (exonal SNPs) that are non-synonymous (nsSNP) were analyzed by (sift, polyphen, Imutant and PHD-snp) softwares, and then SNPs at un-traslated region at 5' ends (5UTR) were analyzed too by SNPs Function prediction software. In this study, Bioinformatics' analysis of MPL gene initiated by SIFTand Polyphen-2server issued to review 197 SNPs and among this SNPs 23 pathological polymorphisms. Among these 23, 20 pathological polymorphisms were found to be very damaging, with higher Polyphen-2score, of the Polyphen-2 server (=1) and SIFT tolerance index of 0.000-0.005. Protein structural analysis was done by modeling of amino acid substitutions using Project Hope. AlsoI-Mutant software used to check their stability and the effect of the native and mutant residues protein and structure for all these pathological polymorphisms. We hope our results will provide useful information that is needed to help researchers to do further studies.
\end{abstract}

Keywords: In silico Analysis, MPL gene, SNPs, SIFT, PolyPhen-2, I-Mutant 3.0 and Project Hope

\section{Introduction}

MPL is a protein in human encoded by (myeloproliferative leukemia protein) or CD110, also known as a Thrombopoietin receptor. [1] The Oncogene was identified in 1990 from the murine myeloproliferative leukemia virus and has the capability of immortalizing bone marrow hematopoietic cells from different lineages. In 1992, the human homologue, named, c-mpl, was cloned. The protein encoded by the c-mpl gene, CD110, is a 635 amino acid transmembrane domain, with two extracellular cytokine receptor domains and two intracellular cytokine receptor box motifs. Sequence data revealed that c-mpl encoded a protein that was homologous with members of the hematopoietic receptor superfamily. Presences of anti-sense oligodeoxynucleotides of c-mpl inhibited megakaryocyte colony formation. TPO-R deficient mice were severely thrombocytopenic, emphasizing the important role of CD110 and Thrombopoietin in megakaryocyte and platelet formation. Upon binding of Thrombopoietin, CD110 is 
dimerized and the JAK family of non-receptor tyrosine kinases, as well as the STAT family, the MAPK family, the adaptor protein $\mathrm{ShC}$ and the receptors themselves become tyrosine phosphorylated [1]. MPL is expressed on early hematopoietic progenitors, megakaryocytes and platelets. Homozygous or compound heterozygous deleterious mutations in the MPL gene lead to congenital megakaryocytic thrombocytopenia (CAMT) [2]. The absence of MPL expression on platelets from CAMT patients has been described in a couple of case studies [3, 4]. However, reduced expression of $M P L$ on platelets has also been demonstrated in patients with other forms of congenital thrombocytopenia, and in patients with inherited or acquired forms of thrombocytosis [5-7]. Mutations in MPL have been classified as being either type I or type II. The type I mutations cause, a complete loss of receptor activity and the type II mutations allow for the maintenance of some receptor function. Patients bearing the type I mutations exhibit bone marrow failure earlier than those with the type II mutations [3-8-9]. Mutations in this gene have been shown to cause familial aplastic anemia. [10] Mutations to this gene are also associated with myelofibrosis and essential thrombocythemia. [11]. In essential thrombocythemia, mutations occur at position 505 or 515. In myelofibrosis, a mutation occurs at position 515 (W515 mutation). These mutations lead to the production of thrombopoietin receptors that are constitutively activated, or constantly turned on, which results in the overproduction of abnormal megakaryocytes [12].

In the present study, we aimed to determine the influence of various polymorphisms in $M P L$ gene on its protein structure that may have an important role in disease susceptibility. The harmful SNPs for the MPL gene have not been predictable to date in silico.

\section{Material and Method}

\subsection{Sequence Datasets and Polymorphism Identification}

MPLgene was investigated in dbSNP/NCBI database NCBI (http://www.ncbi.nlm.nih.gov/snp).197rsSNPs in the coding region were detected. Predictions of deleterious rsSNPs was performed by SIFT and Polyphen-2 software's. Prediction of change in stability due to mutation was performed by I-Mutant 3.0 While prediction of diseaserelated (disease) or neutral polymorphism was performed by PHD-SNP software. The functional impact of the deleterious SNPS was analyzed by project hope. Project hope software was used to highlight the changes occurred because of the deleterious SNPs at the molecular level of the protein 3D structure.

\subsection{Analysis of Functional Consequences of Coding nsSNPs by Sequence Homology Based Method}

\subsubsection{SIFT (Sorting Intolerant from Tolerant)}

Single nucleotide polymorphism (SNP) studies and random mutagenesis projects identify amino acid substitutions in protein-coding regions. Each substitution has the potential to affect protein function. SIFT is a program that predicts by uses sequence homology whether an amino acid substitution affects protein function and hence, potentially alter phenotype. SIFT can distinguish between functionally neutral and deleterious amino acid changes in mutagenesis studies and on human polymorphisms by calculates the probability that an amino acid at a position is tolerated conditional on the most frequent amino acid being tolerated. The cutoff value in the SIFT program is a tolerance index of $\geq 0.05$. If this normalized value is less than a cutoff, the substitution is predicted to be deleterious [13].

\subsubsection{Simulation for Functional Change in Coding rsSNPs by Structure Homology Based Method (PolyPhen-2) (Polymorphism Phenotyping)}

Is an automatic tool for prediction of the possible impact of an amino acid substitution on the structure and function of a human protein. The impact of amino acid allelic variants on protein structure and function can be predicted via analysis of multiple sequence alignments and protein 3D Structures. It Calculates Position-Specific Independent Counts (PSIC) scores for each of the two variants and then computes the PSIC score difference between them. The higher a PSIC score difference the higher functional impact a particular amino acid substitution is likely to have. PolyPhen-2 scores were designated as "probably damaging" (0.95-1), "possibly damaging" (0.7-0.95), and "benign" (0.00-0.31). "Error" (Not enough data to make a prediction [14].

\subsection{Calculation of Stability of Predicted Mutations by Free Energy}

I-Mutant 3.0 suite: Is a suit of Support Vector Machine (SVM) based predictors integrated in an unique web server. It offer the opportunity to predict automatically protein stability change upon single-site mutation starting from protein sequence alone from protein structure when available that calculates protein stability related to a single mutation in units of free energy and also predicts the deleterious SNPs from the human protein sequence [15].

\section{4. (Predictor of Human Deleterious Single Nucleotide Polymorphisms)}

This serveries available at (http://snps.biofold.org/phdsnp/phd-snp.html )

PHD SNP is SVM-based classifier that is optimized to predict if a given single point protein mutation can be classified as disease-related or as neutral polymorphism. The input FASTA sequence of protein along with the residues change was submitted to $\mathrm{PhD}-\mathrm{SNP}$ server for the analysis [16].

\subsection{Modeling rsSNP Locations on Protein Structure}

Modeling by Project hope CMBI (Centre for Molecular and BiomolecularInformatics) is a website where the user can upload the sequence and mutation of interest, HOPE collects 
structural information from a series of sources, including calculations on the $3 \mathrm{D}$ protein structure, sequence annotations in UniProt (The mission of the Universal Protein Resource) (Consortium, 2014) and prediction from the Report of software. HOPE combines this information to give analyses the effect of a certain mutation on the protein structure. HOPE is an online web service where the user can submit a sequence and mutation (Venselaar and CMBI, 2012). or Chimera software (version 1.8) which is a highly extensible program for interactive visualization and analysis of molecular structures and related data [17].

\subsection{Prediction for Gene Prioritization and Predicting Gene Function by GeneMANIA}

GeneMANIA finds other genes that are related to a set of input genes, using a very large set of functional association data which include protein and genetic interactions, pathways, co-expression, co-localization and protein domain similarity. It can use GeneMANIA to find new members of a pathway or complex, find additional genes may have missed in screen or find new genes with a specific function, such as protein kinases [18].

\section{Results}

The SIFT software indicated 23 nsSNPs out of 197 as deleterious i.e. amino acid substitution affects protein function and hence, potentially alter phenotype. The results were shown in Table 1.

Table 1. Results of SIFT score and prediction for MPL gene.

\begin{tabular}{|c|c|c|c|c|}
\hline SNP & Amino Acid Change & Protein ID & SIFT Score & SIFT Prediction \\
\hline rs6088 & E168K & ENSP00000361548 & 0.012 & DELETERIOUS \\
\hline rs28928907 & R102P & ENSP00000361548 & 0 & DELETERIOUS \\
\hline rs28928908 & P275T & ENSP00000361548 & 0.047 & DELETERIOUS \\
\hline rs113696793 & D295G & ENSP00000361548 & 0.018 & DELETERIOUS \\
\hline rs 117656396 & $\mathrm{~L} 265 \mathrm{~F}$ & ENSP00000361548 & 0.013 & DELETERIOUS \\
\hline rs121913611 & $\mathrm{R} 257 \mathrm{C}$ & ENSP00000361548 & 0 & DELETERIOUS \\
\hline rs 137952228 & $\mathrm{~T} 183 \mathrm{M}$ & ENSP00000361548 & 0.014 & DELETERIOUS \\
\hline rs 139770726 & E335K & ENSP00000361548 & 0 & DELETERIOUS \\
\hline rs 141311765 & $\mathrm{Y} 252 \mathrm{H}$ & ENSP00000361548 & 0 & DELETERIOUS \\
\hline rs 145714475 & F245S & ENSP00000361548 & 0 & DELETERIOUS \\
\hline rs 147608148 & S402P & ENSP00000361548 & 0.024 & DELETERIOUS \\
\hline rs 148276667 & $\mathrm{R} 75 \mathrm{H}$ & ENSP00000361548 & 0.018 & DELETERIOUS \\
\hline rs149810307 & V368L & ENSP00000361548 & 0.038 & DELETERIOUS \\
\hline rs200454070 & R426Q & ENSP00000361548 & 0 & DELETERIOUS \\
\hline rs201101813 & D128Y & ENSP00000361548 & 0.002 & DELETERIOUS \\
\hline rs202137992 & P173A & ENSP00000361548 & 0.031 & DELETERIOUS \\
\hline rs202206935 & L109F & ENSP00000361548 & 0.014 & DELETERIOUS \\
\hline rs267598614 & E99K & ENSP00000361548 & 0.032 & DELETERIOUS \\
\hline rs267598615 & E149K & ENSP00000361548 & 0.012 & DELETERIOUS \\
\hline rs369486165 & E332K & ENSP00000361548 & 0.011 & DELETERIOUS \\
\hline rs 371232858 & A418T & ENSP00000361548 & 0.026 & DELETERIOUS \\
\hline rs372164360 & F315I & ENSP00000361548 & 0 & DELETERIOUS \\
\hline rs 373621350 & $\mathrm{R} 390 \mathrm{C}$ & ENSP00000361548 & 0.018 & DELETERIOUS \\
\hline
\end{tabular}

For Polyphen-2 there where, 20 nsSNPs predicted as damaging while three were benign, the damaging SNPs has impact on amino acid allelic variants, protein structure and function. The results were shown in Table 2.

Table 2. Results of Polyphen-2 score and prediction for MPLgene.

\begin{tabular}{lllll}
\hline SNP & Amino Acid Change & Protein ID & Polyphen-2 Score \\
\hline rs6088 & E168K & ENSP00000361548 & 0.994 & Polyphen-2 Prediction \\
rs28928907 & R102P & ENSP00000361548 & 1 & 1 \\
rs28928908 & P275T & ENSP00000361548 & 0.993 & Probably Damaging \\
rs113696793 & D295G & ENSP00000361548 & Probably Damaging \\
rs117656396 & L265F & ENSP00000361548 & Probably Damaging \\
rs121913611 & R257C & ENSP00000361548 & 1 & Benign \\
rs137952228 & T183M & ENSP00000361548 & 0.996 & 1 \\
rs139770726 & E335K & ENSP00000361548 & 0.999 \\
rs141311765 & Y252H & ENSP00000361548 & 0.987 \\
rs145714475 & F245S & ENSP00000361548 & 0.319 \\
rs147608148 & S402P & ENSP00000361548 & 0.999 \\
rs148276667 & R75H & ENSP00000361548 & 0.861 \\
rs149810307 & V368L & ENSP00000361548 & 1 \\
rs200454070 & R426Q & ENSP00000361548 & Probably Damaging Damaging \\
rs201101813 & D128Y & ENSP00000361548 & 0.998 \\
rs202137992 & P173A & ENSP00000361548 & 0.709 \\
rs202206935 & L109F & ENSP00000361548 & 0.085 \\
\hline
\end{tabular}




\begin{tabular}{lllll}
\hline SNP & Amino Acid Change & Protein ID & Polyphen-2 Score & Polyphen-2 Prediction \\
\hline rs267598614 & E99K & ENSP00000361548 & 0.459 & Possibly-Damming \\
rs267598615 & E149K & ENSP00000361548 & 0.753 & Possibly-Damming \\
rs369486165 & E332K & ENSP00000361548 & 0.73 & Possibly-Damming \\
rs371232858 & A418T & ENSP00000361548 & 0.996 & Probably Damaging \\
rs372164360 & F315I & ENSP00000361548 & 0.999 & Probably Damaging \\
rs373621350 & R390C & ENSP00000361548 & 0.992 & Probably Damaging \\
\hline
\end{tabular}

Using I-Mutant software 16 nsSNPs where decreasing the protein stability while 6 nsSNPs were increasing the protein stability. I- Mutant offer the opportunity to predict automatically protein stability change upon single-site mutation. The results were shown in Table 3.

Table 3. Results of I-Mutant prediction, RI and DDG for MPLgene.

\begin{tabular}{|c|c|c|c|c|c|}
\hline SNP & Amino Acid Change & PROTEIN ID & PREDICTION & RI & DDG \\
\hline rs6088 & E168K & ENSP00000361548 & Decreased & 3 & 0.57 \\
\hline rs28928907 & R102P & ENSP00000361548 & Decreased & 1 & 0.42 \\
\hline rs 28928908 & $\mathrm{P} 275 \mathrm{~T}$ & ENSP00000361548 & Decreased & 4 & 0.61 \\
\hline rs113696793 & D295G & ENSP00000361548 & Decreased & 9 & 1.91 \\
\hline rs117656396 & L265F & ENSP00000361548 & Decreased & 6 & 0.55 \\
\hline rs121913611 & $\mathrm{R} 257 \mathrm{C}$ & ENSP00000361548 & Decreased & 4 & 0.6 \\
\hline rs137952228 & $\mathrm{T} 183 \mathrm{M}$ & ENSP00000361548 & Increased & 1 & 0.1 \\
\hline rs 139770726 & E335K & ENSP00000361548 & Decreased & 5 & 1.29 \\
\hline rs141311765 & Y252H & ENSP00000361548 & Decreased & 0 & 0.46 \\
\hline rs 145714475 & F245S & ENSP00000361548 & Decreased & 4 & 0.47 \\
\hline rs148276667 & $\mathrm{R} 75 \mathrm{H}$ & ENSP00000361548 & Decreased & 5 & 0.5 \\
\hline rs 149810307 & V368L & ENSP00000361548 & Increased & 2 & 0.1 \\
\hline rs200454070 & R426Q & ENSP00000361548 & Decreased & 7 & 1.5 \\
\hline rs201101813 & D128Y & ENSP00000361548 & Increased & 7 & 0.33 \\
\hline rs202137992 & P173A & ENSP00000361548 & Decreased & 3 & 0.12 \\
\hline rs202206935 & L109F & ENSP00000361548 & Increased & 2 & 0.03 \\
\hline rs267598614 & E99K & ENSP00000361548 & Decreased & 6 & 1.8 \\
\hline rs267598615 & E149K & ENSP00000361548 & Decreased & 3 & 0.62 \\
\hline rs369486165 & E332K & ENSP00000361548 & Increased & 4 & 0.25 \\
\hline rs371232858 & $\mathrm{A} 418 \mathrm{~T}$ & ENSP00000361548 & Decreased & 9 & 1.37 \\
\hline rs 372164360 & F315I & ENSP00000361548 & Increased & 0 & 0.71 \\
\hline rs373621350 & $\mathrm{R} 390 \mathrm{C}$ & ENSP00000361548 & Decreased & 6 & 0.59 \\
\hline
\end{tabular}

To predict the capability of causing disease or being neutral, PHD software was used the results were show in Table 4 .

Table 4. Results of PHD prediction, RI and Probability for MPLgene.

\begin{tabular}{llllll}
\hline SNP & Amino Acid Change & Protein ID & Prediction & RI & Probability \\
\hline rs6088 & E168K & ENSP00000361548 & Disease & 3 & 0.646 \\
rs28928907 & R102P & ENSP00000361548 & Disease & 6 & 0.797 \\
rs28928908 & P275T & ENSP00000361548 & Disease & 1 & 0.46 \\
rs113696793 & D295G & ENSP00000361548 & Disease & 5 & 0.7 \\
rs117656396 & L265F & ENSP00000361548 & Neutral & 7 & 0.14 \\
rs121913611 & R257C & ENSP00000361548 & Disease & 3 & 0.659 \\
rs137952228 & T183M & ENSP00000361548 & Neutral & 5 & 0.232 \\
rs139770726 & E335K & ENSP00000361548 & Neutral & 0 & 0.497 \\
rs141311765 & Y252H & ENSP00000361548 & Disease & 0 & 0.502 \\
rs145714475 & F245S & ENSP00000361548 & Disease & 0 & 0.455 \\
rs148276667 & R75H & ENSP00000361548 & Disease & 3 & 0.674 \\
rs149810307 & V368L & ENSP00000361548 & Neutral & 4 & 0.316 \\
rs200454070 & R426Q & ENSP00000361548 & Neutral & 5 & 0.236 \\
rs201101813 & D128Y & ENSP00000361548 & Disease & 3 & 0.654 \\
rs202137992 & P173A & ENSP00000361548 & Disease & 2 & 0.606 \\
rs202206935 & L109F & ENSP00000361548 & Neutral & 3 & 0.361 \\
rs267598614 & E99K & ENSP00000361548 & Disease & 3 & 0.637 \\
rs267598615 & E149K & ENSP00000361548 & Disease & 1 & 0.567 \\
rs369486165 & E332K & ENSP00000361548 & Neutral & 6 & \\
rs371232858 & A418T & ENSP00000361548 & Neutral & 3 & 0.184 \\
rs372164360 & F315I & ENSP00000361548 & Neutral & 2 & 0.372 \\
rs373621350 & R390C & ENSP00000361548 & Disease & 1 & 0.399 \\
\hline
\end{tabular}

Classification the nsSNPs to disease and neutral through the SNP \& Go prediction tool revealed 20 nsSNPs as Neutral and only one was as disease related nsSNP (rs28928907/ R102P). The table 5 below showed the results 
Table 5. Results of SNP \& GO prediction, RI and Probability for MPLgene.

\begin{tabular}{llllll}
\hline SNP & Amino Acid Change & Protein ID & Prediction & RI & Probability \\
\hline rs6088 & E168K & ENSP00000361548 & Neutral & 5 & 0.262 \\
rs28928907 & R102P & ENSP00000361548 & Disease & 1 & 0.53 \\
rs28928908 & P275T & ENSP00000361548 & Neutral & 9 & 0.054 \\
rs113696793 & D295G & ENSP00000361548 & Neutral & 1 & 0.465 \\
rs117656396 & L265F & ENSP00000361548 & Neutral & 9 & 0.026 \\
rs121913611 & R257C & ENSP00000361548 & Neural & 5 & 0.275 \\
rs137952228 & T183M & ENSP00000361548 & Neutral & 9 & 0.06 \\
rs139770726 & E335K & ENSP00000361548 & Neutral & 8 & 0.099 \\
rs141311765 & Y252H & ENSP00000361548 & Neutral & 8 & 0.098 \\
rs148276667 & R75H & ENSP00000361548 & Neutral & 3 & 0.355 \\
rs149810307 & V368L & ENSP00000361548 & Neutral & 8 & 0.086 \\
rs200454070 & R426Q & ENSP00000361548 & Neutral & 7 & 0.134 \\
rs201101813 & D128Y & ENSP00000361548 & Neutral & 0 & 0.48 \\
rs202137992 & P173A & ENSP00000361548 & Neutral & 8 & 0.124 \\
rs202206935 & L109F & ENSP00000361548 & Neutral & 8 & 0.093 \\
rs267598614 & E99K & ENSP00000361548 & Neutral & 4 & 0.289 \\
rs267598615 & E149K & ENSP00000361548 & Neutral & 5 & 0.245 \\
rs369486165 & E332K & ENSP00000361548 & Neutral & 9 & 0.049 \\
rs371232858 & A418T & ENSP00000361548 & Neutral & 9 & 0.071 \\
rs372164360 & F315I & ENSP00000361548 & Neutral & 8 & 0.119 \\
rs373621350 & R390C & ENSP00000361548 & Neutral & \\
\hline
\end{tabular}

\section{Project-Hope results}

The prediction of the mutation of Arginine into a Proline at position 102. (rs28928907) was shown in Figure 1. Each amino acid has its own specific size, charge, and hydrophobicity-value. The original wild-type residue and newly introduced mutant residue often differ in these properties. The mutant residue is smaller than the wild-type residue. The wild-type residue charge was POSITIVE; the mutant residue charge is NEUTRAL. The wild-type residue is more hydrophobic than the mutant residue<smiles>N=C(N)NCCCC(N)C(=O)O</smiles>

Arginine

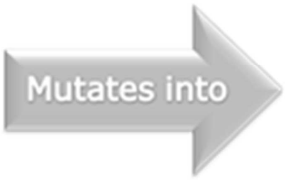

Proline

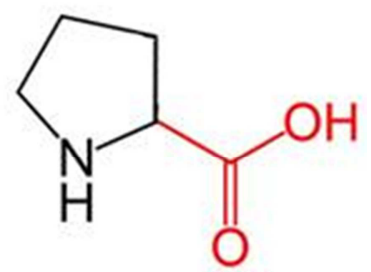

Figure 1. The schematic structures of the original (left) and the mutant (right) amino acid. The backbone, which is the same for each amino acid, is colored red. The side chain, unique for each amino acid, is colored black.

For the prediction of the mutation P275T (rs28928908), each amino acid has its own specific size, charge, and hydrophobicity-value. The original wild-type residue and newly introduced mutant residue often differ in these properties. The wild-type residue is more hydrophobic than the mutant residue. The results were shown in Figure 2.
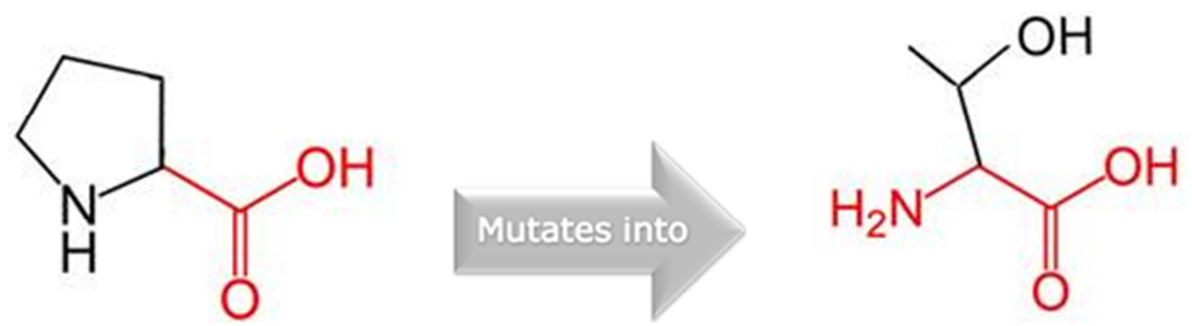

Figure 2. The schematic structures of the original (left) and the mutant (right) amino acid. The backbone, which is the same for each amino acid, is colored red. The side chain, unique for each amino acid, is colored black.

Using GeneMANIA software it predicts the associated genes with MPL gene, results were shown in Figure 3 and the coexpressed and shared domain with, MPL gene, results were shown in Table 6. 


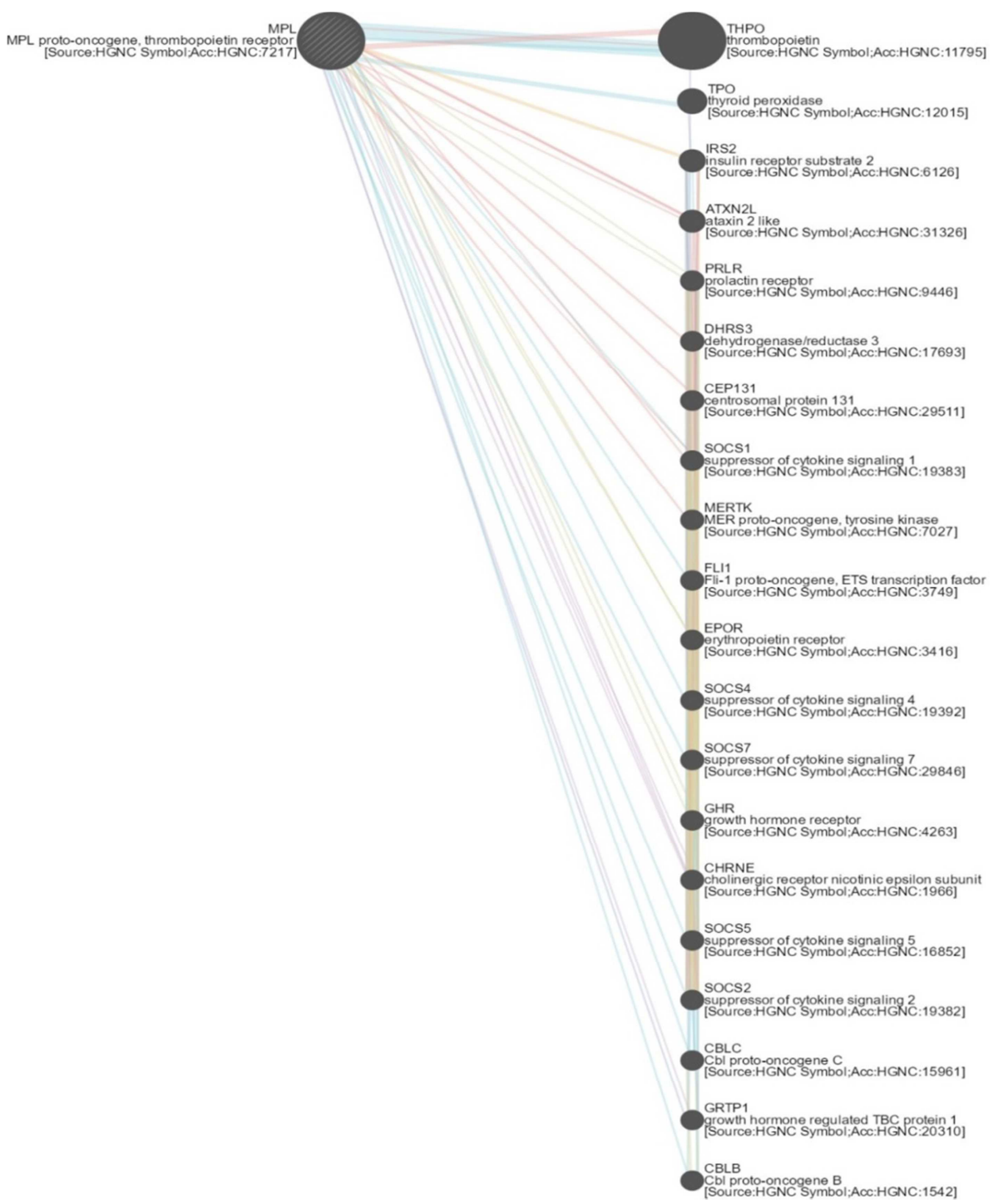

Figure 3. GeneMANIA showing the MPL and associated genes.

Table 6. The co-expressed and shared domain with MPL gene by GeneMANIA.

\begin{tabular}{llll}
\hline Gene & Description & Co Expression & Shared Domain \\
\hline MPL & MPLproto-oncogene, thrombopoietinreceptor [Source: HGNCSymbol; Acc: HGNC: 7217] & & \\
THPO & thrombopoietin [Source: HGNC Symbol; Acc: HGNC: 11795] & No & No \\
TPO & thyroid peroxidase [Source: HGNC Symbol; Acc: HGNC: 12015] & Yes & No \\
IRS2 & insulin receptor substrate 2 [Source: HGNC Symbol; Acc: HGNC: 6126] & Yes & No \\
ATXN2L & ataxin 2 like [Source: HGNC Symbol; Acc: HGNC: 31326] & Yes & No \\
PRLR & prolactin receptor [Source: HGNC Symbol; Acc: HGNC: 9446] & Yes & Yes \\
DHRS3 & dehydrogenase/reductase 3 [Source: HGNC Symbol; Acc: HGNC: 17693] & Yes & No \\
CEP131 & centrosomal protein 131 [Source: HGNC Symbol; Acc: HGNC: 29511] & Yes & Yes \\
SOCS1 & suppressor of cytokine signaling 1 [Source: HGNC Symbol; Acc: HGNC: 19383] & Yes & No \\
MERTK & MER proto-oncogene, tyrosine kinase [Source: HGNCSymbol; Acc: HGNC: 7027] & Yes & No \\
FLI1 & Fli-1 proto-oncogene, ETS transcription factor [Source: HGNC Symbol; Acc: HGNC: 3749 ] & Yes & Yes \\
EPOR & erythropoietin receptor [Source: HGNC Symbol; Acc: HGNC: 3416] & Yes & Yes \\
SOCS4 & suppressor of cytokine signaling 4 [Source: HGNC Symbol; Acc: HGNC: 19392] & No & Yes \\
SOCS7 & suppressor of cytokine signaling 7 [Source: HGNC Symbol; Acc: HGNC: 29846] & Yes & Yes \\
GHR & growth hormone receptor [Source: HGNC Symbol; Acc: HGNC: 4263] & Yes & No \\
CHRNE & Cholinergic receptor nicotinic epsilon subunit [Source: HGNCSymbol; Acc: HGNC: 1966] & Yes & Yes \\
SOCS5 & suppressor of cytokine signaling 5 [Source: HGNC Symbol; Acc: HGNC: 16852] & Yes & Yes \\
SOCS2 & suppressor of cytokine signaling 2 [Source: HGNC Symbol; Acc: HGNC: 19382] & NO \\
CBLC & Cbl proto-oncogene C [Source: HGNC Symbol; Acc: HGNC: 15961] & Yes & Nes \\
GRTP1 & growth hormone regulated TBC protein 1 [Source: HGNCSymbol; Acc: HGNC: 20310] & NO \\
CBLB & Cbl proto-oncogene B [Source: HGNC Symbol; Acc: HGNC: 1542] & Yes \\
\hline
\end{tabular}




\section{Discussion}

MPL gene has a vital role in human body and it is coexpressed with 16 genes and shared domain with 10 genes. $M P L$ gene was investigated in NCBI database (http://www.ncbi.nlm.nih.gov/). It contains 2399 SNPswith197SNPs in the coding region. Non synonymous SNPs were analyzed by SIFT software, and only 23 SNPs predicted to be deleterious. These deleterious SNPs were analyzed using Polyphen-2 software to predict the damaging SNPs; only 20 SNPs were predicted to be deleterious in both software. I-mutant software was used to evaluate the degree of stability due to mutation. The present study found that 16 SNPs were scored to decrease the stability of the protein, While only seven SNPs had increased in the stability of the protein. Moreover, $\mathrm{PhD}$ was another promising software used beside the I-Mutant, 12 SNPs were sorted as a disease related, while 10 SNPs were neutral, but there was one SNP obtained no result from the database (rs147608148). For more confirmation for the mutation the study used SNP \& Go software that only one SNP (rs28928907) was reported as a disease related, while the others SNPs were reported as a neutral. The Project Hope software was used to detect the effect the mutation on the protein structure and function many of the SNPs found were affecting these properties as illustrated by rs28928907 and rs28928908 as shown in figure 1and 2. Moreover a study done in 2011 suggested that $M P L$ $\mathrm{W} 515 \mathrm{~K} / \mathrm{L}$ was negative ET cases, and no mutations detected previously proposed as potential candidate drivers [19], and this findings might suggest why this SNPs was not detected as a pathogenic SNPs in this study, so another candidates SNPs or mutations may be studied as a target in the future for the ET cases. The quest for the full complement of driver mutations in ET therefore remains open.

\section{Conclusions}

The MPL gene plays important roles in cell signaling and proliferation. Mutations in this gene confer constitutive activation of the JAK-STAT pathway and other pathways promoting differentiation and proliferation of different lineage. The available data from the NCBI dbSNP database for $M P L$ gene has been analyzed through several SNP analyzing tools and the predicted deleterious SNPs were evaluated for their deleterious effect on the protein function and stability. The study concluded that only one nsSNP which is the Arginine into a Proline at position 102. (rs28928907) is of high value to be suggested as diagnostic nsSNP for $M P L$ gene.

\section{References}

[1] Elsayed AG, Ranavaya A, Jamil MO. MPL Y252H anMd PL F126fs mutations in essential thrombocythemia: Case series and review of literature. Hematology reports. 2019 Feb 19; 11 (1).

[2] Vainchenker W, Plo I, Marty C, Varghese LN, Constantinescu
SN. The role of the thrombopoietin receptor MPL in myeloproliferative neoplasms: recent findings and potential therapeutic applications. Expert review of hematology. 2019 Jun 3; 12 (6): 437-48.

[3] Ballmaier M, Germeshausen M, Schulze H, Cherkaoui K, Lang S, Gaudig A, Krukemeier S, Eilers M, Strauß G, Welte K. C-mpl mutations are the cause of congenital amegakaryocytic thrombocytopenia. Blood. 2001 Jan 1; 97 (1): 139-46.

[4] Savoia A, Dufour C, Locatelli F, Noris P, Ambaglio C, Rosti V, Zecca M, Ferrari S, Di Bari F, Corcione A, Di Stazio M. Congenital amegakaryocytic thrombocytopenia: clinical and biological consequences of five novel mutations. haematologica. 2007 Sep 1; 92 (9): 1186-93.

[5] Heller PG, Glembotsky AC, Gandhi MJ, Cummings CL, Pirola CJ, Marta RF, Kornblihtt LI, Drachman JG, Molinas FC. Low Mpl receptor expression in a pedigree with familial platelet disorder with predisposition to acute myelogenous leukemia and a novel AML1 mutation. Blood. 2005 Jun 15; 105 (12): 4664-70.

[6] Horikawa Y, Matsumura I, Hashimoto K, Shiraga M, Kosugi S, Tadokoro S, Kato T, Miyazaki H, Tomiyama Y, Kurata Y, Matsuzawa Y. Markedly reduced expression of platelet c-mpl receptor in essential thrombocythemia. Blood. 1997 Nov 15; 90 (10): 4031-8.

[7] Li J, Xia Y, Kuter DJ. The platelet thrombopoietin receptor number and function are markedly decreased in patients with essential thrombocythaemia. British journal of haematology. 2000 Dec; 111 (3): 943-53.

[8] King S, Germeshausen M, Strauss G, Welte K, Ballmaier M. Congenital amegakaryocytic thrombocytopenia: a retrospective clinical analysis of 20 patients. British journal of haematology. 2005 Dec; 131 (5): 636-44.

[9] Germeshausen M, Ballmaier M, Welte K. MPL mutations in 23 patients suffering from congenital amegakaryocytic thrombocytopenia: the type of mutation predicts the course of the disease. Human mutation. 2006 Mar; 27 (3): 296-283.

[10] Walne AJ, Dokal A, Plagnol V, Beswick R, Kirwan M, de la Fuente J, Vulliamy T, Dokal I. Exome sequencing identifies MPL as a causative gene in familial aplastic anemia. Haematologica. 2012 Apr 1; 97 (4): 524-8.

[11] Tefferi A, Lasho TL, Finke CM, Knudson RA, Ketterling R, Hanson CH, Maffioli M, Caramazza D, Passamonti F, Pardanani A. CALR vs JAK2 vs MPL-mutated or triplenegative myelofibrosis: clinical, cytogenetic and molecular comparisons. Leukemia. 2014 Jul; 28 (7): 1472.

[12] Tefferi A. Novel mutations and their functional and clinical relevance in myeloproliferative neoplasms: JAK2, MPL, TET2, ASXL1, CBL, IDH and IKZF1. Leukemia. 2010 Jun; 24 (6): 1128.

[13] González-Pérez A, López-Bigas N. Improving the assessment of the outcome of nonsynonymous SNVs with a consensus deleteriousness score, Condel. The American Journal of Human Genetics. 2011 Apr 8; 88 (4): 440-9.

[14] Capriotti E, Fariselli P, Calabrese R, Casadio R. Predicting protein stability changes from sequences using support vector machines. Bioinformatics. 2005 Jan 1; 21 (suppl_2): ii54-8. 
[15] Källberg M, Wang H, Wang S, Peng J, Wang Z, Lu H, Xu J. Template-based protein structure modeling using the RaptorX web server. Nature protocols. 2012 Aug; 7 (8): 1511.

[16] Warde-Farley D, Donaldson SL, Comes O, Zuberi K, Badrawi R, Chao P, Franz M, Grouios C, Kazi F, Lopes CT, Maitland A. The GeneMANIA prediction server: biological network integration for gene prioritization and predicting gene function. Nucleic acids research. 2010 Jun 21; 38 (suppl_2): W214-20.
[17] Santos LC, Ribeiro JC, Silva NP, Cerutti J, Silva MR, Chauffaille MD. Cytogenetics, JAK2 and MPL mutations in polycythemia vera, primary myelofibrosis and essential thrombocythemia. Revista brasileira de hematologia e hemoterapia. 2011 Dec; 33 (6): 417-24.

[18] Feener EP, Rosario F, Dunn SL, Stancheva Z, Myers MG. Tyrosine phosphorylation of Jak2 in the JH2 domain inhibits cytokine signaling. Molecular and cellular biology. 2004 Jun 1; 24 (11): 4968-78. 